\title{
CSF Cytokines in Aging, Multiple Sclerosis, and Dementia
}

\author{
William T. Hu ${ }^{1,2 \star}$, Jennifer Christina Howell ${ }^{1,3}$, Tugba Ozturk ${ }^{1}$, Umesh Gangishetti ${ }^{1}$, \\ Alexander L. Kollhoff ${ }^{1}$, Jaime M. Hatcher-Martin ${ }^{4}$, Albert M. Anderson ${ }^{5}$ and \\ William R. Tyor ${ }^{1,6}$
}

\begin{abstract}
${ }^{1}$ Department of Neurology, Emory University, Atlanta, GA, United States, ${ }^{2}$ Center for Neurodegenerative Disease, Emory University, Atlanta, GA, United States, ${ }^{3}$ Alzheimer's Disease Research Center, Emory University, Atlanta, GA, United States, ${ }^{4}$ Jean and Paul Amos Parkinson's Disease and Movement Disorders Program, Emory University, Atlanta, GA, United States, ${ }^{5}$ Department of Internal Medicine, Emory University, Atlanta, GA, United States, ${ }^{6}$ Atlanta VA Medical Center, Decatur, GA, United States
\end{abstract}

\section{OPEN ACCESS}

Edited by:

Stefan Bittner,

Johannes Gutenberg University

Mainz, Germany

Reviewed by:

Anna Fogdell-Hahn,

Karolinska Institute (KI), Sweden

Olaf Stuve,

University of Texas Southwestern Medical Center,

United States

*Correspondence:

William T. Hu

wthu@emory.edu:

william.hu@emory.edu

Specialty section: This article was submitted to

Multiple Sclerosis and

Neuroimmunology,

a section of the journal

Frontiers in Immunology

Received: 15 October 2018

Accepted: 21 February 2019

Published: 15 March 2019

Citation:

Hu WT, Howell JC, Ozturk T,

Gangishetti $U$, Kollhoff AL,

Hatcher-Martin JM, Anderson AM and Tyor WR (2019) CSF Cytokines in

Aging, Multiple Sclerosis, and

Dementia. Front. Immunol. 10:480.

doi: 10.3389/fimmu.2019.00480
Inflammation is a common process involved in aging, multiple sclerosis (MS), and age-related neurodegenerative disorders such as Alzheimer's disease (AD) and Parkinson's disease (PD), but there is limited evidence for the effects of aging on inflammation in the central nervous system. We collected cerebrospinal fluid (CSF) from 105 healthy control subjects representing a wide age range (23-86), and analyzed levels of cytokines associated innate immunity (TNF- $\alpha$ ) and different T-helper subtypes: interferon-gamma induced protein 10 (IP-10) for Th1, interleukin-10 (IL-10) for Th2, and interleukin 8 (IL-8/CXCL8) for Th17. We show that CSF levels of TNF- $\alpha$, IP-10, and IL-8 all increased linearly with age, but levels of IL-10 demonstrated a U-shaped relationship with age. We further found greater age-related increases in TNF- $\alpha, I L-10$, and IL-8 relative to increases in IP-10 levels, consistent with a shift from Th1 to other inflammatory phenotypes. Finally, when we analyzed the same four cytokines in people with neurological disorders, we found that MS and $A D$, but not PD or dementia with Lewy bodies, further accentuated the age-related shift from Th1- to non-Th1-related cytokines. We propose that CSF cytokine levels represent powerful surrogates of brain inflammation and aging, and some, but not all, neurological disorders accelerate the shift away from Th1 phenotypes.

Keywords: inflammaging, neuroinflammation and neurodegeneration, IP10, IL10, IL8, Alzheimer's disease, Parkinsons disease (PD), dementia with Lewy bodies (DLB)

\section{INTRODUCTION}

Neuro-inflammation is increasingly implicated in age-related neurodegenerative diseases such as Alzheimer's disease (AD) and Parkinson's disease (PD) $(1,2)$. Following examples in multiple sclerosis (MS) and other demyelinating conditions, many recent human neurodegenerative studies have analyzed soluble cytokine levels in the blood or cerebrospinal fluid (CSF) to generate hypotheses testable in other cohorts or animal models (3-5). However, factors such as age can 
themselves bias the immune system and therefore cytokine levels, including aging-associated pro-inflammatory bias and a shift from Th1 to Th2/innate immunity dominance $(6,7)$. These patterns, usually sterile (free of symptomatic infection), independent of organ- or system-based disease, and involving the innate and adaptive immune systems, have been together referred to as inflamm-aging $(8,9)$. Potential drivers for inflamm-aging include persistent low-grade viral and bacterial infection, changing gut microbiome (10), and clearance of accumulating self-proteins. Because of inflamm-aging, it is not always straightforward to distinguish between the effects of disease and non-disease factors on absolute cytokine levels. A thorough evaluation of disease-associated cytokine alterations thus requires an adequate number of healthy subjects across a wide age range to detect not only cytokine level differences but also multi-cytokine profiles, which inform about disease specific changes that also reflect complex immune pathways.

Because recruiting healthy subjects to undergo CSF collection can be difficult, multiple smaller cohorts may need to be combined to generate a larger cohort. This process is not without its own challenges, including the need for standardized CSF collection across multiple research centers with diseasespecific protocols $(11,12)$, reproducible cytokine quantitation, and data-sharing. Over the past 4 years, we have recruited 105 healthy control (HC) subjects over a wide age-range (2286) to participate in multiple disease-oriented studies using a modified but standardized Alzheimer's Disease Neuro-Imaging (ADNI) protocol (12). This cohort provides a unique opportunity to simultaneously interrogate CSF cytokines from different functional pathways across seven decades of life. Here we measured four cytokines associated with innate immunity and helper T cell subtypes (Th1, Th2, Th17) in these HC subjects, and present a new framework to assess imbalance between cytokine pairs in central nervous system (CNS) disorders including MS, $\mathrm{AD}, \mathrm{PD}$, and dementia with Lewy bodies (DLB).

\section{MATERIALS AND METHODS}

\section{Standard Protocol Approvals and Patient Consents}

This study was carried out in accordance to US Code of Federal Regulations Title 45 Part 46 Protection of Human Subjects, and Emory University and Emory School of Medicine policies. The protocols were approved by the Emory University Institutional Review Board. Banked CSF samples were used for this study, and all subjects had previously given written informed consent according to the Declaration of Helsinki for long-term sample storage and future analysis.

\section{Subject Characterization}

Older $\mathrm{HC}$ and $\mathrm{AD}$ subjects $(n=52$, median age 69 , range 48-89) were recruited during a previous study on CSF and MRI biomarkers of aging and dementia in Caucasian and African Americans (13). Younger HC subjects were recruited from the Emory Cognitive Neurology Clinic, Emory Alzheimer's
Disease Research Center, and Emory University in an ongoing study of pre-symptomatic carriers for dominantlyinherited frontotemporal lobar degeneration, and a separate study examining CSF biomarkers of HIV. All HC subjects underwent detailed neuropsychological testing (14) to confirm normal cognition. Older and younger HC subjects were then combined to form a continuum (median age 60; range 22$105)$, with $52 / 105$ (50\%) HC subjects younger than 60 years of age. MS $(n=18$, median age 48 , range $28-74), \operatorname{PD}(n=37$, median age 69 , range $41-81)$, and DLB $(n=23$, median age 68 , range 47-80) were recruited from the Emory Neuroimmunology, Movement Disorders, and Cognitive Neurology Clinics. Because recruitment was completed prior to the latest revisions in diagnostic criteria for MS and DLB in 2017, all MS patients were diagnosed according to the 2010 revised McDonald criteria (15), and all DLB patients were diagnosed according to the 2005 McKeith criteria (16). PD patients had clinical features and findings consistent with the Movement Disorders Society Parkinson's disease criteria (17).

\section{CSF Collection}

CSF samples were all previously collected using a modified Alzheimer's Disease Neuroimaging Initiative protocol at Emory University (18). Briefly, CSF was collected into $15 \mathrm{~mL}$ polypropylene tubes via a 24-gauge atraumatic needle and syringe aspiration without overnight fasting. CSF in polypropylene tubes was immediately inverted several times, aliquotted $(500 \mu \mathrm{L})$, labeled, and frozen at $-80^{\circ} \mathrm{C}$ until analysis. CSF samples from 13 young $\mathrm{HC}$ subjects (mean age 37.0, range 23-54) were centrifuged at 2,500 $\mathrm{rpm}$ after collection before freezing. We previously carried out a prospective experiment centrifuging in this condition half of freshly collected CSF in 16 subjects, and compared levels of eight CSF cytokines (including the four included in the current study) in the supernatant with levels from the uncentrifuged halves. We showed that centrifugation did not influence measured cytokine levels (19), and these samples can be analyzed together. We have also determined the stability of each analyte through freeze-thawing following a pre-established protocol such that the measured levels from frozen samples most closely reflect in vivo levels (18).

\section{CSF Cytokine Analysis}

Four inflammatory proteins were selected for their preferential association with innate immunity or different immune cell populations, including tumor necrosis alpha (TNF- $\alpha)$ (20), Th-2 related interleukin 10 (IL-10) (21), and Th17-related interleukin 8 (IL-8/CXCL8) (22). Levels of Th1-associated interferon gamma were not consistently detectable, and a downstream marker interferon gamma-induced protein (IP-10/CXCL10, Th1) (23) was used instead as a surrogate. Assays were performed in a Luminex 200 platform using the Merck-Milliplex MAP Human Cytokine Panel (HCYTOMAG-60K, Merck-Millipore, Burlington, MA) following the manufacturer's protocol except two $100 \mu \mathrm{L}$ aliquots of CSF were used for duplicates instead of what was stated in the protocol. IL-9 is also associated with Th17 pathways but its CSF alterations are challenging to interpret because of influence from race and potentially other factors 


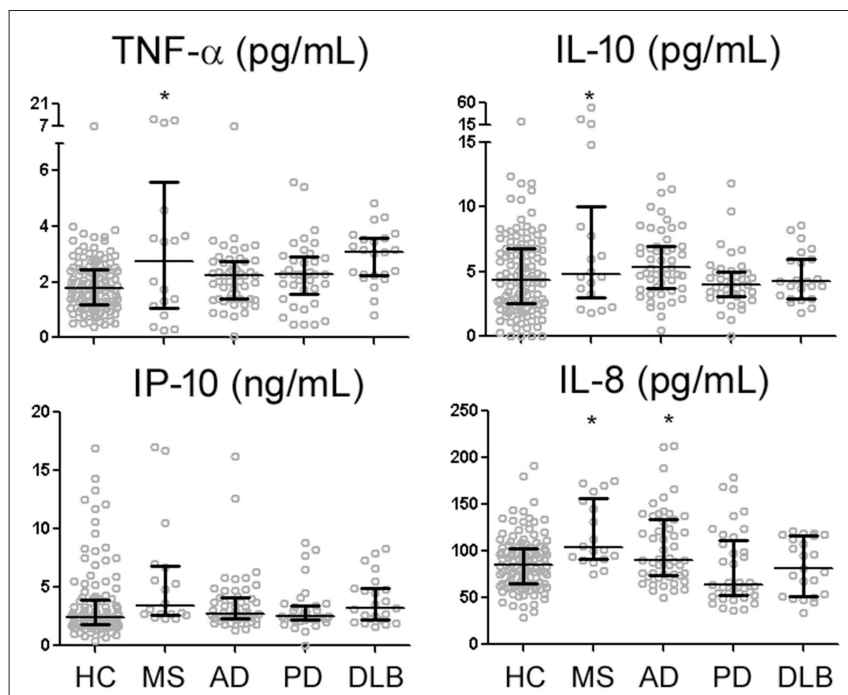

FIGURE 1 | CSF levels of four reliably-measured cytokines in healthy control subjects (HC), multiple sclerosis (MS), Alzheimer's disease (AD), Parkinson's disease (PD), and dementia with Lewy bodies (DLB). ${ }^{*} p<0.05$ compared to $\mathrm{HC}$.

(Wharton and $\mathrm{Hu}$, unpublished data). Analysis involving IL9 was thus deferred here. IL- 6 was also not measured because its CSF levels were found to be normal in multiple previous studies including in aging, AD (5, 24, 25), and MS (26-29), potentially confounded by the large inter-individual variability (30). Intermediate precision achieved in our laboratory using thirteen biological replicates (different aliquots from the same lumbar puncture for thirteen subjects) over 9 weeks was $4.8 \%$ for IP-10, 9.4\% for TNF- $\alpha, 16.5 \%$ for IL-10, and $12.0 \%$ for IL-8.

\section{Statistical Analysis}

HC subjects were analyzed with age as a continuous variable or according to categories each spanning two decades of life: 20-39, 40-59, and 60-79. Six subjects older than 80 (range 8186) were combined with the last group to form the category of $\geq 60$. For basic demographic comparison, Chi-squared tests and analysis of variance were used to determine categorical and continuous baseline variables across the five diagnostic categories (HC, MS, AD, PD, DLB). CSF IP-10 levels did not have a normal distribution, and were thus $\log _{10}$-transformed. Analysis of co-variance (ANCOVA) was used to analyze the main effect of diagnosis on CSF cytokine levels, adjusting for age and sex. Pearson's correlational analysis was used to determine the association between cytokines. Regression analysis was used to determine the impact of inflamm-aging in $\mathrm{HC}$ subjects with $\log _{10}$-transformed IP-10, age, and gender, and the interaction between age and $\log _{10}(\mathrm{IP}-10)$ as variables.

To determine impact of neurological disorders on inflammaging, levels of $\log _{10}$ (IP-10), TNF- $\alpha$, IL-10, and IL-8 were Z-transformed to adjust for absolute level and distribution differences between the cytokines. Specifically, mean and standard deviation values were calculated for each cytokine from all HC subjects, and all subjects' cytokine levels were then converted to Z-scores. Regression analysis was then used to determine the impact of disease on inflamm-aging, with $\log _{10}$ (IP10 ), age, gender, age $\mathrm{X} \log _{10}(\mathrm{IP}-10)$, and diagnosis $\mathrm{X} \log _{10}$ (IP-10) as variables.

\section{RESULTS}

Among 105 HC subjects, CSF levels of TNF- $\alpha$, IL-10, and IP-10 all strongly correlated with one another ( $\mathrm{R}$ range of $0.354-0.449, p$ $<0.001)$, while IL-8 only correlated with IP-10 levels $(R=0.469$, $p<0.001)$. Univariate analysis showed higher levels of TNF- $\alpha$, IP-10, IL-10, and IL-8 in MS than HC (Figure 1). In contrast, univariate analysis identified $\mathrm{AD}$ as the only non-MS disorder associated with higher levels of a single cytokine (IL-8) than HC (Figure 1). Regression analysis including HC and MS patients taking into account age, gender, MS diagnosis, and MS treatment showed MS treatment to be associated with relative decrease in IL-10 levels $(p=0.014)$.

Because inflamm-aging can potentially complicate the interpretation of group-level comparisons, we next explored the effects of age on CSF cytokine levels in HC subjects using regression analysis. Consistent with a pro-inflammatory bias, TNF- $\alpha(R=0.250, p=0.010)$, IP-10 $\left(R=0.453\right.$ for $\log _{10^{-}}$ transformed values, $p<0.001)$ and IL-8 $(R=0.439, p<0.001)$ levels all increased with age (Table 1, Figure 2) while controlling for gender. Interestingly, instead of a linear or step-wise increase, we observed a trend of lower CSF IL-10 levels in the middle-aged group (40-59) than the older and younger groups (Table 1). This led us to model this $U$-shaped relationship between age and IL-10 levels using a quadratic equation (Figure 2, $R=0.495, p$ $<0.001$ ), with the inflection point (lowest levels) at 50 years of age. ANCOVA showed that MS was associated with greater IP-10 $\left[F_{(2,123)}=14.537, p<0.001\right]$, TNF- $\alpha[F=19.321, p<0.001]$, IL-10 $[F=18.921, p<0.001]$, and IL-8 $[F=23.358, p<0.001]$ levels (Figure 2), but only IP-10 and IL-8 increased with age in MS ( $p<0.001$ for both).

Beyond paralleled increases in cytokine levels beyond the age of 50, we further analyzed if age modified the relationship between Th1-associated IP-10 and other cytokines by examining the interaction between age and IP-10. This showed that, not only is there an age-associated increase in these CSF cytokines, age further accentuated the positive correlation between IP-10 and TNF- $\alpha$, IL-10, and IL- 8 levels $(p<0.001$ for the interaction terms, Table 2) after controlling for gender. These findings suggest relatively greater increases in these three cytokines for each standard unit of age-associated increase in IP-10, in keeping with a consistent phenotype switch from Th1 to innate immunity (31) and Th2 (7) phenotypes.

While inflammatory changes are either pathogenic (e.g., MS) or commonly observed in neurodegenerative disorders (e.g., AD, $\mathrm{PD}$ ), it is not clear if each neurological disorder can specifically alter the balance between cytokines linked to inflamm-aging. We thus analyzed if IP-10 levels interacted with disease status in addition to its interaction with age (Figure 3) by examining if each disease alters the slope linking IP-10 and the three other cytokines. We found that, for each standard deviation increase 
TABLE 1 | Basic demographic and CSF cytokine levels of people with healthy control $(\mathrm{HC})$ with normal cognition according to age group as well as multiple sclerosis (MS).

$$
\begin{array}{lccc}
\text { MS }(n=18) & \text { NC, 20-39 } & \text { NC, 40-59 } & \text { NC, } \geq 60 \\
\text { year }(n=23) & \text { year }(n=29) & \text { year }(n=53)
\end{array}
$$

\begin{tabular}{|c|c|c|c|c|}
\hline Male & $4(22 \%)$ & 16 (70\%) & $11(38 \%)$ & 24 (45\%) \\
\hline Age (SD), year & $50.0(12.8)$ & $31.9(4.2)$ & $52.8(5.8)$ & $69.5(6.6)$ \\
\hline $\begin{array}{l}\text { Disease duration } \\
\text { (SD), yr }\end{array}$ & $11.0(12.9)$ & - & - & - \\
\hline \multicolumn{5}{|l|}{ MS Type } \\
\hline RRMS & 15 & & & \\
\hline PPMS & 2 & & & \\
\hline SPMS & 1 & & & \\
\hline $\begin{array}{l}\text { Current MS } \\
\text { therapy }\end{array}$ & 8 & & & \\
\hline Interferon $\beta$-1a & 2 & & & \\
\hline $\begin{array}{l}\text { Glatiramer } \\
\text { acetate }\end{array}$ & 2 & & & \\
\hline Fingolimod & 2 & & & \\
\hline $\begin{array}{l}\text { Dimethyl } \\
\text { fumarate }\end{array}$ & 1 & & & \\
\hline Natalizumab & 1 & & & \\
\hline TNF- $\alpha$ (SD), pg/mL & $4.55(5.78)^{\star}$ & $1.51(0.76)$ & $1.75(1.28)$ & $2.11(0.89)$ \\
\hline IP-10 (SD), pg/mL & $5.61(4.64)^{\star \star}$ & $2.46(2.71)$ & 3.09 (3.38) & $4.07(2.65)$ \\
\hline IL-10 (SD), pg/mL & $9.51(12.20)^{\star}$ & $4.57(2.48)$ & $2.82(1.87)$ & $5.30(2.93)$ \\
\hline IL-8 (SD), pg/mL & $119.1(35.2)^{\star}$ & $67.5(22.9)^{\dagger}$ & 88.4 (21.3) & $96.4(31.5)$ \\
\hline
\end{tabular}

RRMS, relapsing-remitting MS; PPMS, primary progressive MS; SPMS, secondary progressive MS.

*Higher in MS than other groups.

**Higher in MS than 22-39 and 40-59 year old groups.

${ }^{+}$Lower in the youngest group than the other two.

in IP-10 beyond the age-associated effect on IP-10, both MS ( $p<$ $0.001)$ and $\mathrm{AD}(p=0.026)$ were associated with greater change in TNF- $\alpha$ than HC, AD was associated with greater change in IL-10 levels $(p=0.018)$ than HC, and MS was associated with greater change in IL- 8 levels $(p=0.013)$ than HC. Importantly, these interactions were independent of the age-associated shift. While PD and DLB were associated with greater TNF- $\alpha$ levels and lower IL-8 levels than NC, these disorders did not modify the inflamm-aging relationships between IP-10 and other cytokines. Combining subjects with PD and DLB as Lewy body disease did not change the outcomes.

\section{DISCUSSION}

Inflamm-aging is known to occur in the blood $(32,33)$ and bone marrow (34), but only limited evidence exists in the human CNS $(35,36)$. Here we show in a cross-sectional cohort spanning seven decades of life that CSF levels of cytokines associated with innate immunity, Th1, Th2, and Th17 all increased with age. We further show that these cytokines did not increase according to age at the same rates, and aging was associated with greater relative increases in cytokines levels linked to innate immunity, Th2, and Th17 than Th1. Finally, we show that MS and AD further

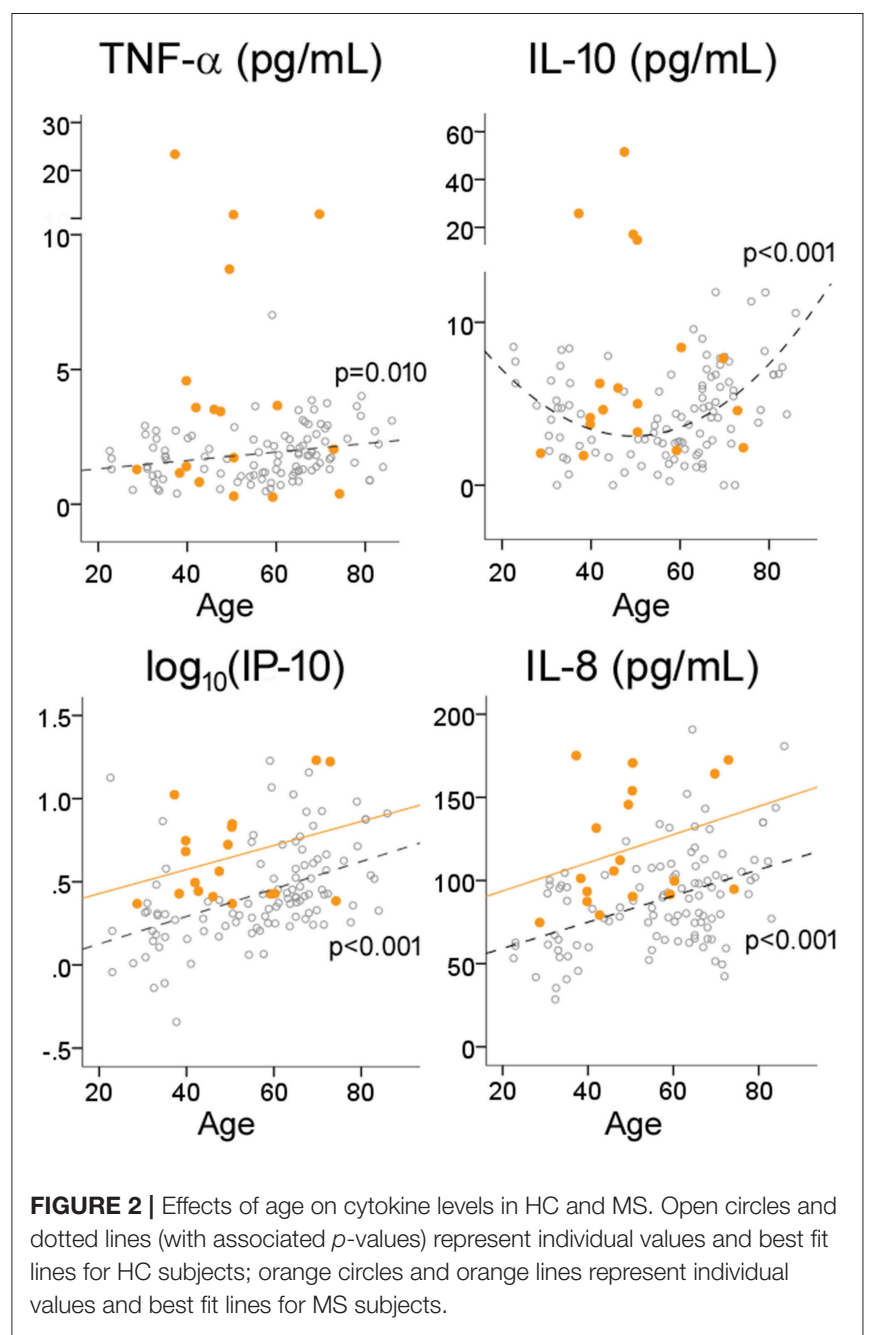

TABLE 2 | Age modifies the relationship between IP-10 and other cytokines in HC (coefficients and $p$-values are shown).

\begin{tabular}{lccc}
\hline & TNF- $\alpha$ & IL-10 & IL-8 \\
\hline Age, year & $-0.007(p=0.357)$ & $-0.052(p=0.053)$ & $-6.259(p=0.207)$ \\
Male gender & $0.133(p=0.456)$ & $0.955(p=0.047)$ & $-6.838(p=0.161)$ \\
$\log _{10}(\mathrm{IP}-10)$ & $0.287(p=0.780)$ & $-3.985(p=0.146)$ & $-4.000(p=0.888)$ \\
Age $X \log _{10}(\mathrm{IP}-10)$ & $0.023(p<0.001)$ & $0.146(p=0.003)$ & $0.759(p<0.001)$
\end{tabular}

accentuate the age-associated phenotype shift from Th1 to nonTh1 pathways independent of inflamm-aging. Altogether, these CSF findings provide evidence for inflamm-aging in the CNS, and put forth the hypothesis that some-but not all-neurological disorders mimic key features of inflamm-aging.

Inflamm-aging is thought to result from chronic unresolved infection and immunosenesence among other factors (37). There is a general paucity of evidence for inflamm-aging in the human CNS due to limited availability of normal post-mortem brain tissue across the life span $(36,38,39)$. Using brains from 57 neuropathologically and cognitively normal people from seven 


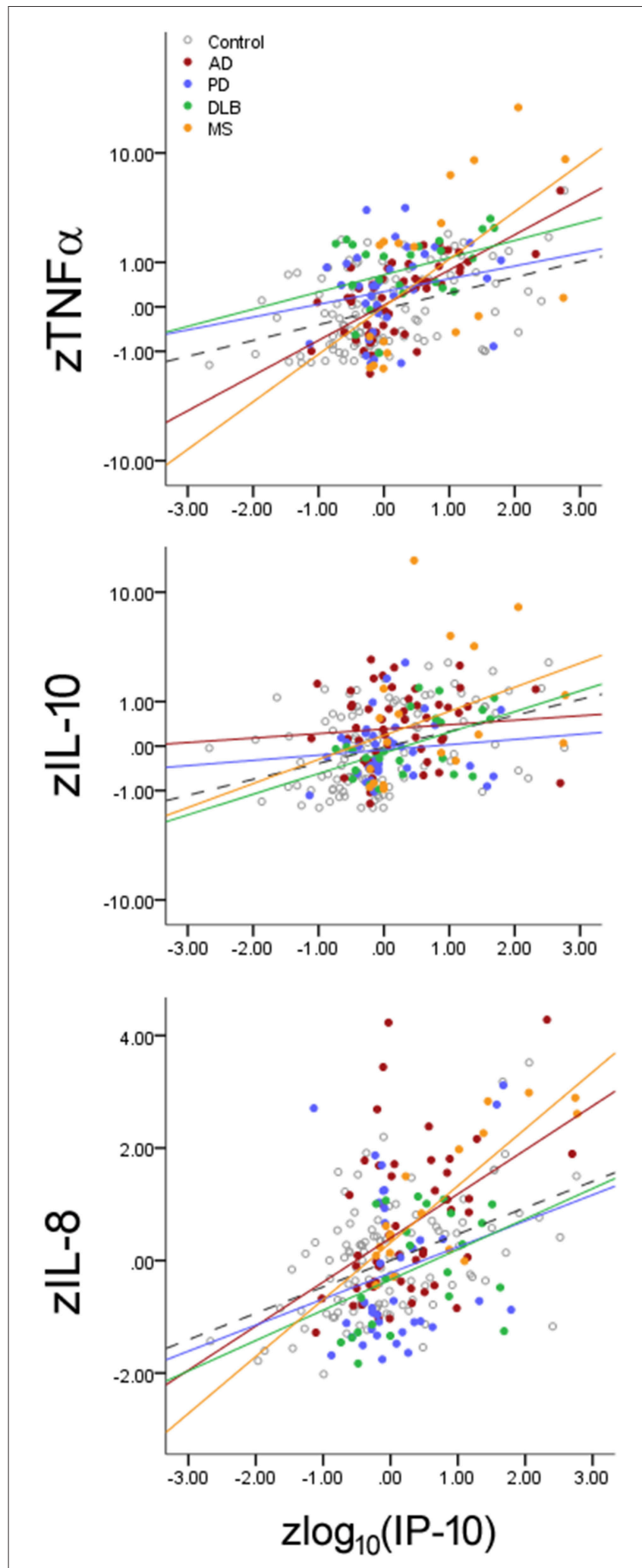

FIGURE 3 | Effects of neurological diseases on the relationship between Th1-associated IP-10 and cytokines associated with innate immunity (TNF- $\alpha$ ), Th2 (IL-10), and Th17 (IL-8). Cytokine levels were normalized according to HC

(Continued)
FIGURE 3 I mean and standard deviation values for illustrative purposes. Accounting for inflamm-aging showed that MS and AD enhanced the relationship between these cytokines, while PD and DLB changed the absolute cytokine levels without altering the relationships. Dotted lines represent best fit line for $\mathrm{HC}$ subjects.

brain banks, an expression analysis showed aging and $\mathrm{AD}$ to each upregulate genes associated the complement pathway and the toll-like receptors (39). They also identified brain IL-10 as a cytokine implicated in inflamm-aging, in keeping with our findings of age- and $\mathrm{AD}$-associated increase in IL-10 relative to IP-10 elevations. However, a difference in brain IL-10 expression was not reported by a subsequent RNA-Seq study examining 79 subjects when cases were analyzed according to age (under 60 or over 60) (36), possibly due to the U-shaped distribution of CSF IL-10 levels according to age. This age-dependent U-shaped curve can also potentially account for inconsistent findings of normal $(28,40,41)$ or elevated $(29,42)$. CSF IL-10 levels in MS patients from cross-sectional studies. At the same time, the aging-associated trough for CSF IL-10 levels in the fifth decade may identify a window during which the brain is susceptible to immune-mediated processes. Because the peak age at onset for MS precedes this natural IL-10 decline by one to two decades, it is conceivable that patients with MS may experience this IL-10 trough-and the associated unopposed increase in TNF- $\alpha$, IP-10, and IL-8-earlier in life. Indeed, age at onset has prognostic roles in initial phenotype, progression, and disability (43-45), and the CSF cytokine milieu at onset may better represent biological age with respects to neuroinflammation than chronological age. While definitive testing of this hypothesis would require presymptomatic identification of MS patients, healthy young adults at increased risks for MS through family history or a GWASbased polygenic risk score (46) may determine candidate genes associated with premature IL-10 decline.

Increased CSF TNF- $\alpha$, IP-10, and IL-8 levels have been previously reported in untreated and treated MS (29, 47-51). A treatment-associated decrease in IL-10 levels in MS may seem contradictory at first especially if premature IL-10 decline may be a risk factor for MS. Previous studies have shown coupled increase of CSF TNF- $\alpha$ and IL-10 during MS flare-ups (52) as well as their coupled normalization after interferon- $\beta$ or natalizumab treatment $(53,54)$. Therefore, lower IL-10 levels in treated vs. untreated MS patients likely reflect generalized reduction in immune activation, and MS-treatment does not attenuate the bias toward IL-10 from IP-10. It remains to be determined whether individual cytokine levels or the relative balance between cytokines better predict disease progression or response to therapy.

The exact mechanisms underlying inflamm-aging are not clear. Relevant to the CNS, aging is associated with dendritic remodeling (55), reduced CSF outflow (56), increasing risks of tau-related pathology (57), premature immunosenescense (58), and often white matter hyperintensity associated with cerebrovascular disease on MRI. While we cannot clearly distinguish between CSF cytokine changes due to chronological 
aging, immunosenescence, and cumulative subclinical injury common in aging (e.g., ischemia, hypoxia), our work-to the best of our knowledge-provides the first CSF-based evidence for CNS inflamm-aging. Major strengths of the current study include the pooling of subjects with $\mathrm{HC}$ and neurological disorders based on standardized collection protocols across multiple sites, and the joint analysis of cytokine levels with age-adjusted balance between cytokines. At the expense of scope, we restricted our analysis to four CSF analytes for their readily detectable levels (e.g., CSF IFN- $\gamma$ and IL-4 are not easily detected in most CSF samples), measurement accuracy (e.g., some commercial assays fail to validate when non-kit protein standards are used), high intermediate precision (reproducibility between assays run on different days), stability during processing (e.g., freezethawing), and invariance across racial groups. Omitting cytokine measures that fall short on specificity or reproducibility allowed for more consistent statistical analysis to address inflammaging and to detect linear as well as the U-shaped trends. It is important to note that the cross-sectional nature of our study is suggestive-not confirmatory-of an age-related change over time. An independent validation cohort and longitudinal analysis within the same individuals are necessary to confirm these findings. We also did not quantify different cell types in the CSF, and immunophenotyping will be necessary to test whether cytokine level changes are due to cell type switch, hyperactivity, or both. Because of our focus on CNS inflammaging, we did not measure plasma cytokines because we have previously found little correlation between plasma and CSF cytokine levels in $\mathrm{HC}$ or people with neurological diseases. In keeping with this, prior studies reported dissociated patterns of plasma and CSF cytokine alterations in MS, but a comparative study of peripheral and CNS inflamm-aging may bring about

\section{REFERENCES}

1. Lapenna A, De Palma M, Lewis CE. Perivascular macrophages in health and disease. Nat Rev Immunol. (2018) 18:689-702. doi: 10.1038/s41577-018-0056-9

2. Skaper SD, Facci L, Zusso M, Giusti P. An Inflammation-centric view of neurological disease: beyond the neuron. Front Cell Neurosci. (2018) 12:72. doi: $10.3389 /$ fncel.2018.00072

3. Ray S, Britschgi M, Herbert C, Takeda-Uchimura Y, Boxer A, Blennow K, et al. Classification and prediction of clinical Alzheimer's diagnosis based on plasma signaling proteins. Nat Med. (2007) 13:1359-62. doi: 10.1038/nm1653

4. Craig-Schapiro R, Perrin RJ, Roe CM, Xiong C, Carter D, Cairns NJ, et al. YKL-40: a novel prognostic fluid biomarker for preclinical Alzheimer's disease. Biol Psychiatry. (2010) 68:903-12. doi: 10.1016/j.biopsych.2010.08.025

5. Hu WT, Chen-Plotkin A, Arnold SE, Grossman M, Clark CM, Shaw LM, et al. Novel CSF biomarkers for Alzheimer's disease and mild cognitive impairment. Acta Neuropathol. (2010) 119:669-78. doi: 10.1007/s00401-010-0667-0

6. Franceschi C, Bonafe M, Valensin S, Olivieri F, De Luca M, Ottaviani E, et al. Inflamm-aging. An evolutionary perspective on immunosenescence. Ann N Y Acad Sci. (2000) 908:244-54. doi: 10.1111/j.1749-6632.2000.tb06651.x

7. Sandmand M, Bruunsgaard H, Kemp K, Andersen-Ranberg K, Pedersen AN, Skinhoj $\mathrm{P}$, et al. Is ageing associated with a shift in the balance between Type 1 and Type 2 cytokines in humans? Clin Exp Immunol. (2002) 127:107-14.

8. Franceschi C, Garagnani P, Parini P, Giuliani C, Santoro A. Inflammaging: a new immune-metabolic viewpoint for age-related diseases. Nat Rev Endocrinol. (2018) 14:576-90. doi: 10.1038/s41574-018-0059-4 additional insight. Nevertheless, we present a new framework for simultaneously evaluating CSF cytokines related to inflammaging and neurological disorders that goes beyond up- or downregulation, and extends the Th1 to non-Th1 shift associated with aging to $\mathrm{AD}$.

\section{DATA AVAILABILITY}

The raw data supporting the conclusions of this manuscript will be made available by the authors, without undue reservation, to any qualified researcher.

\section{AUTHOR CONTRIBUTIONS}

$\mathrm{WH}, \mathrm{JH}$, and UG contributed to conception and design of the study. WH, JH, TO, UG, AK, JH-M, AA, and WT organized the dataset. $\mathrm{WH}$ performed the statistical analysis. $\mathrm{WH}$ and $\mathrm{JH}$ wrote sections of the manuscript. TO, UG, JH-M, AA, and WT contributed to the revision of the manuscript. All authors read and approved the submitted version.

\section{FUNDING}

This study was sponsored by the NIH K23AG042856 (WH), R21AG043885 (WH), R01AG054046 (WH), K23MH095679 (AA), R01MH116695 (WT), I01BX01506 (WT), the Patterson Family Foundation, and the Bumpus Foundation (JH-M).

\section{ACKNOWLEDGMENTS}

We would like to thank Drs. Jonathan D. Glass, Stewart A. Factor, and Neil S. Lava for their assistance in recruitment.

9. Rea IM, Gibson DS, McGilligan V, McNerlan SE, Alexander HD, Ross OA. Age and age-related diseases: role of inflammation triggers and cytokines. Front Immunol. (2018) 9:586. doi: 10.3389/fimmu.2018.00586

10. Biagi E, Nylund L, Candela M, Ostan R, Bucci L, Pini E, et al. Through ageing, and beyond: gut microbiota and inflammatory status in seniors and centenarians. PLoS ONE. (2010) 5:e10667. doi: 10.1371/journal.pone.0010667

11. Freedman MS, Thompson EJ, Deisenhammer F, Giovannoni G, Grimsley G, Keir G, et al. Recommended standard of cerebrospinal fluid analysis in the diagnosis of multiple sclerosis: a consensus statement. Arch Neurol. (2005) 62:865-70. doi: 10.1001/archneur.62.6.865

12. Hu WT, Watts KD, Shaw LM, Howell JC, Trojanowski JQ, Basra S, et al. CSF beta-amyloid 1-42 - what are we measuring in Alzheimer's disease? Ann Clin Transl Neurol. (2015) 2:131-9. doi: 10.1002/acn3.160

13. Howell JC, Parker MW, Watts KD, Kollhoff A, Tsvetkova DZ, Hu WT. Research lumbar punctures among African Americans and Caucasians: perception predicts experience. Front Aging Neurosci. (2016) 8:296. doi: 10.3389/fnagi.2016.00296

14. Howell JC, Watts KD, Parker MW, Wu J, Kollhoff A, Wingo TS, et al. Race modifies the relationship between cognition and Alzheimer's disease cerebrospinal fluid biomarkers. Alzheimers Res Ther. (2017) 9:88. doi: 10.1186/s13195-017-0315-1

15. Polman CH, Reingold SC, Banwell B, Clanet M, Cohen JA, Filippi M, et al. Diagnostic criteria for multiple sclerosis: 2010 revisions to the McDonald criteria. Ann Neurol. (2011) 69:292-302. doi: 10.1002/ana.22366

16. McKeith IG, Dickson DW, Lowe J, Emre M, O’Brien JT, Feldman $\mathrm{H}$, et al. Diagnosis and management of dementia with Lewy bodies: 
third report of the DLB Consortium. Neurology. (2005) 65:1863-72. doi: 10.1212/01.wnl.0000187889.17253.b1

17. Postuma RB, Berg D, Stern M, Poewe W, Olanow CW, Oertel W, et al. MDS clinical diagnostic criteria for Parkinson's disease. Mov Disord. (2015) 30:1591-601. doi: 10.1002/mds.26424

18. Hu WT, Watts K, Grossman M, Glass J, Lah JJ, Hales C, et al. Reduced CSF p-Tau181 to Tau ratio is a biomarker for FTLD-TDP. Neurology. (2013) 81:1945-52. doi: 10.1212/01.wnl.0000436625.63650.27

19. Gangishetti U, Christina Howell J, Perrin RJ, Louneva N, Watts KD, Kollhoff A, et al. Non-beta-amyloid/tau cerebrospinal fluid markers inform staging and progression in Alzheimer's disease. Alzheimers Res Ther. (2018) 10:98. doi: $10.1186 / \mathrm{s} 13195-018-0426-3$

20. Clark IA. How TNF was recognized as a key mechanism of disease. Cytokine Growth Factor Rev. (2007) 18:335-43. doi: 10.1016/j.cytogfr.2007.04.002

21. Hunt NH, Grau GE. Cytokines: accelerators and brakes in the pathogenesis of cerebral malaria. Trends Immunol. (2003) 24:491-9. doi: 10.1016/S1471-4906(03)00229-1

22. Pelletier M, Maggi L, Micheletti A, Lazzeri E, Tamassia N, Costantini C, et al. Evidence for a cross-talk between human neutrophils and Th17 cells. Blood. (2010) 115:335-43. doi: 10.1182/blood-2009-04-216085

23. Haabeth OA, Lorvik KB, Hammarstrom C, Donaldson IM, Haraldsen G, Bogen $\mathrm{B}$, et al. Inflammation driven by tumour-specific Thl cells protects against B-cell cancer. Nat Commun. (2011) 2:240. doi: 10.1038/ncomms1239

24. Schuitemaker A, Dik MG, Veerhuis R, Scheltens P, Schoonenboom $\mathrm{NS}$, Hack $\mathrm{CE}$, et al. Inflammatory markers in $\mathrm{AD}$ and $\mathrm{MCI}$ patients with different biomarker profiles. Neurobiol Aging. (2009) 30:1885-9. doi: 10.1016/j.neurobiolaging.2008.01.014

25. Craig-Schapiro R, Kuhn M, Xiong C, Pickering EH, Liu J, Misko TP, et al. Multiplexed immunoassay panel identifies novel CSF biomarkers for Alzheimer's disease diagnosis and prognosis. PLoS ONE. (2011) 6:e18850. doi: 10.1371/journal.pone.0018850

26. Uzawa A, Mori M, Ito M, Uchida T, Hayakawa S, Masuda S, et al. Markedly increased CSF interleukin-6 levels in neuromyelitis optica, but not in multiple sclerosis. J Neurol. (2009) 256:2082-4. doi: 10.1007/s00415-009-5274-4

27. Horellou P, Wang M, Keo V, Chretien P, Serguera C, Waters P, et al. Increased interleukin-6 correlates with myelin oligodendrocyte glycoprotein antibodies in pediatric monophasic demyelinating diseases and multiple sclerosis. $J$ Neuroimmunol. (2015) 289:1-7. doi: 10.1016/j.jneuroim.2015.10.002

28. Matejcikova Z, Mares J, Prikrylova Vranova H, Klosova J, Sladkova V, Dolakova J, et al. Cerebrospinal fluid inflammatory markers in patients with multiple sclerosis: a pilot study. J Neural Transm. (2015) 122:273-7. doi: 10.1007/s00702-014-1244-9

29. Sosvorova L, Kanceva R, Vcelak J, Kancheva L, Mohapl M, Starka L, et al. The comparison of selected cerebrospinal fluid and serum cytokine levels in patients with multiple sclerosis and normal pressure hydrocephalus. Neuro Endocrinol Lett. (2015) 36:564-71. doi: 10.1046/j.1365-2249.2002.01736.x

30. Brosseron F, Krauthausen M, Kummer M, Heneka MT. Body fluid cytokine levels in mild cognitive impairment and Alzheimer's disease: a comparative overview. Mol Neurobiol. (2014) 50:534-44. doi: 10.1007/s12035014-8657-1

31. Panda A, Arjona A, Sapey E, Bai F, Fikrig E, Montgomery RR, et al. Human innate immunosenescence: causes and consequences for immunity in old age. Trends Immunol. (2009) 30:325-33. doi: 10.1016/j.it.2009.05.004

32. Bartlett DB, Firth CM, Phillips AC, Moss P, Baylis D, Syddall H, et al. The age-related increase in low-grade systemic inflammation (Inflammaging) is not driven by cytomegalovirus infection. Aging Cell. (2012) 11:912-5. doi: $10.1111 /$ j.1474-9726.2012.00849.x

33. Nevalainen T, Kananen L, Marttila S, Jylha M, Hervonen A, Hurme M, et al. Transcriptomic and epigenetic analyses reveal a gender difference in aging-associated inflammation: the vitality $90+$ study. Age. (2015) 37:9814. doi: 10.1007/s11357-015-9814-9

34. Pangrazzi L, Meryk A, Naismith E, Koziel R, Lair J, Krismer M, et al. Inflammaging influences immune cell survival factors in human bone marrow. Eur J Immunol. (2017) 47:481-92. doi: 10.1002/eji.201646570

35. Rhinn $\mathrm{H}$, Abeliovich A. Differential aging analysis in human cerebral cortex identifies variants in TMEM106B and GRN that regulate aging phenotypes. Cell Syst. (2017) 4:404-15.e405. doi: 10.1016/j.cels. 2017.02.009
36. Cheng H, Xuan H, Green CD, Han Y, Sun N, Shen H, et al. Repression of human and mouse brain inflammaging transcriptome by broad genebody histone hyperacetylation. Proc Natl Acad Sci USA. (2018) 115:7611-6. doi: $10.1073 /$ pnas. 1800656115

37. Franceschi C, Campisi J. Chronic inflammation (inflammaging) and its potential contribution to age-associated diseases. J Gerontol A Biol Sci Med Sci. (2014) 69(Suppl. 1):S4-9. doi: 10.1093/gerona/glu057

38. Berchtold NC, Cribbs DH, Coleman PD, Rogers J, Head E, Kim R, et al. Gene expression changes in the course of normal brain aging are sexually dimorphic. Proc Natl Acad Sci USA. (2008) 105:15605-10. doi: $10.1073 /$ pnas. 0806883105

39. Cribbs DH, Berchtold NC, Perreau V, Coleman PD, Rogers J, Tenner AJ, et al. Extensive innate immune gene activation accompanies brain aging, increasing vulnerability to cognitive decline and neurodegeneration: a microarray study. J Neuroinflammation. (2012) 9:179. doi: 10.1186/1742-2094-9-179

40. Fainardi E, Rizzo R, Melchiorri L, Vaghi L, Castellazzi M, Marzola A, et al. Presence of detectable levels of soluble HLA-G molecules in CSF of relapsingremitting multiple sclerosis: relationship with CSF soluble HLA-I and IL10 concentrations and MRI findings. J Neuroimmunol. (2003) 142:149-58. doi: 10.1016/S0165-5728(03)00266-2

41. Braitch M, Nunan R, Niepel G, Edwards LJ, Constantinescu CS. Increased osteopontin levels in the cerebrospinal fluid of patients with multiple sclerosis. Arch Neurol. (2008) 65:633-5. doi: 10.1001/archneur.65.5.633

42. Maxeiner HG, Marion Schneider E, Kurfiss ST, Brettschneider J, Tumani H, Bechter K. Cerebrospinal fluid and serum cytokine profiling to detect immune control of infectious and inflammatory neurological and psychiatric diseases. Cytokine. (2014) 69:62-7. doi: 10.1016/j.cyto.2014.05.008

43. Scalfari A, Neuhaus A, Daumer M, Ebers GC, Muraro PA. Age and disability accumulation in multiple sclerosis. Neurology. (2011) 77:1246-52. doi: 10.1212/WNL.0b013e318230a17d

44. Cossburn M, Ingram G, Hirst C, Ben-Shlomo Y, Pickersgill TP, Robertson NP. Age at onset as a determinant of presenting phenotype and initial relapse recovery in multiple sclerosis. Mult Scler. (2012) 18:45-54. doi: $10.1177 / 1352458511417479$

45. Roy S, Frndak S, Drake AS, Irwin L, Zivadinov R, Weinstock-Guttman B, et al. Differential effects of aging on motor and cognitive functioning in multiple sclerosis. Mult Scler. (2017) 23:1385-93. doi: 10.1177/1352458516679036

46. Sawcer S. The major cause of multiple sclerosis is environmental: genetics has a minor role-no. Mult Scler. (2011) 17:1174-5. doi: $10.1177 / 1352458511421106$

47. Maimone D, Gregory S, Arnason BG, Reder AT. Cytokine levels in the cerebrospinal fluid and serum of patients with multiple sclerosis. $J$ Neuroimmunol. (1991) 32:67-74. doi: 10.1016/0165-5728(91)90073-G

48. Sorensen TL, Tani M, Jensen J, Pierce V, Lucchinetti C, Folcik VA, et al. Expression of specific chemokines and chemokine receptors in the central nervous system of multiple sclerosis patients. J Clin Invest. (1999) 103:807-15. doi: $10.1172 /$ JCI5150

49. Narikawa K, Misu T, Fujihara K, Nakashima I, Sato S, Itoyama Y. CSF chemokine levels in relapsing neuromyelitis optica and multiple sclerosis. J Neuroimmunol. (2004) 149:182-6. doi: 10.1016/j.jneuroim. 2003.12.010

50. Rossi S, Motta C, Studer V, Barbieri F, Buttari F, Bergami A, et al. Tumor necrosis factor is elevated in progressive multiple sclerosis and causes excitotoxic neurodegeneration. Mult Scler. (2014) 20:304-12. doi: $10.1177 / 1352458513498128$

51. de Flon P, Soderstrom L, Laurell K, Dring A, Sundstrom P, Gunnarsson $\mathrm{M}$, et al. Immunological profile in cerebrospinal fluid of patients with multiple sclerosis after treatment switch to rituximab and compared with healthy controls. PLoS ONE. (2018) 13:e0192516. doi: 10.1371/journal. pone. 0192516

52. Rodriguez-Sainz Mdel C, Sanchez-Ramon S, de Andres C, Rodriguez-Mahou M, Munoz-Fernandez MA. Th1/Th2 cytokine balance and nitric oxide in cerebrospinal fluid and serum from patients with multiple sclerosis. Eur Cytokine Netw. (2002) 13:110-4.

53. Khademi M, Wallstrom E, Andersson M, Piehl F, Di Marco R, Olsson T. Reduction of both pro- and anti-inflammatory cytokines after 6 months of interferon beta-1a treatment of multiple sclerosis. J Neuroimmunol. (2000) 103:202-10. doi: 10.1016/S0165-5728(99)00184-8 
54. Mellergard J, Edstrom M, Vrethem M, Ernerudh J, Dahle C. Natalizumab treatment in multiple sclerosis: marked decline of chemokines and cytokines in cerebrospinal fluid. Mult Scler. (2010) 16:208-17. doi: $10.1177 / 1352458509355068$

55. Mrak RE, Griffin ST, Graham DI. Aging-associated changes in human brain. $J$ Neuropathol Exp Neurol. (1997) 56:1269-75. doi: 10.1097/00005072-19971200000001

56. Ma Q, Ineichen BV, Detmar M, Proulx ST. Outflow of cerebrospinal fluid is predominantly through lymphatic vessels and is reduced in aged mice. Nat Commun. (2017) 8:1434. doi: 10.1038/s41467017-01484-6

57. Crary JF, Trojanowski JQ, Schneider JA, Abisambra JF, Abner EL, Alafuzoff I, et al. Primary age-related tauopathy (PART): a common pathology associated with human aging. Acta Neuropathol. (2014) 128:755-66. doi: 10.1007/s00401-014-1349-0

58. Thewissen M, Linsen L, Somers V, Geusens P, Raus J, Stinissen P. Premature immunosenescence in rheumatoid arthritis and multiple sclerosis patients. Ann N Y Acad Sci. (2005) 1051:255-62. doi: 10.1196/annals.13 61.066
Conflict of Interest Statement: WH has a patent on the use of CSF biomarkers to diagnose FTLD-TDP (US 9,618,522); has received research support from Avid Radiopharmaceuticals (Philadelphia, PA) and Fujirebio US (Malvern, PA); serves as a consultant to AARP, Inc, Locks Law Firm, Interpleader Law, and Roche Diagnostics USA, ViveBio LLC; has received travel support from HoffmanLaRoche and Abbvie. The funder played no role in the study design, the collection, analysis or interpretation of data, the writing of this paper or the decision to submit it for publication.

The remaining authors declare that the research was conducted in the absence of any commercial or financial relationships that could be construed as a potential conflict of interest.

Copyright (C) 2019 Hu, Howell, Ozturk, Gangishetti, Kollhoff, Hatcher-Martin, Anderson and Tyor. This is an open-access article distributed under the terms of the Creative Commons Attribution License (CC BY). The use, distribution or reproduction in other forums is permitted, provided the original author(s) and the copyright owner(s) are credited and that the original publication in this journal is cited, in accordance with accepted academic practice. No use, distribution or reproduction is permitted which does not comply with these terms. 